\title{
Optics of water cloud droplets mixed with black-carbon aerosols
}

\author{
Michael I. Mishchenko, ${ }^{1, *}$ Li Liu, ${ }^{1,2}$ Brian Cairns, ${ }^{1}$ and Daniel W. Mackowski ${ }^{3}$ \\ ${ }^{1}$ NASA Goddard Institute for Space Studies, 2880 Broadway, New York, New York 10025, USA \\ ${ }^{2}$ Department of Applied Physics and Applied Mathematics, Columbia University, 2880 Broadway, New York, New York 10025, USA \\ ${ }^{3}$ Department of Mechanical Engineering, Auburn University, Auburn, Alabama 36849, USA \\ ${ }^{*}$ Corresponding author: michael.i.mishchenko@nasa.gov
}

Received March 6, 2014; revised March 20, 2014; accepted March 25, 2014;

posted March 25, 2014 (Doc. ID 207767); published April 21, 2014

\begin{abstract}
We use the recently extended superposition $T$-matrix method to calculate scattering and absorption properties of micrometer-sized water droplets contaminated by black carbon. Our numerically exact results reveal that, depending on the mode of soot-water mixing, the soot specific absorption can vary by a factor exceeding 6.5. The specific absorption is maximized when the soot material is quasi-uniformly distributed throughout the droplet interior in the form of numerous small monomers. The range of mixing scenarios captured by our computations implies a wide range of remote sensing and radiation budget implications of the presence of black carbon in liquid-water clouds. We show that the popular Maxwell-Garnett effective-medium approximation can be used to calculate the optical cross sections, single-scattering albedo, and asymmetry parameter for the quasi-uniform mixing scenario, but is likely to fail in application to other mixing scenarios and in computations of the elements of the scattering matrix. (c) 2014 Optical Society of America
\end{abstract}

OCIS codes: (290.0290) Scattering; (290.4020) Mie theory; (290.4210) Multiple scattering; (290.5850) Scattering, particles.

http://dx.doi.org/10.1364/OL.39.002607

It is widely recognized that contamination by black carbon may cause substantial changes in the optical and radiative properties of liquid-water clouds, with strong implications for Earth's climate system (see, e.g., Refs. [1-3] and references therein). However, accurate quantification of these changes had been a very challenging problem since direct computer solutions of the Maxwell equations for realistic models of micrometer-sized droplets with various types of soot impurities were impracticable $[4,5]$. Instead, the computations of electromagnetic scattering by such particles had been based on the simplified model of a droplet with only one inclusion [1] or on approximate approaches, such as various effective-medium theories (EMTs) $[\underline{2}, \underline{3}, \underline{6}]$ and the Monte Carlo ray-tracing technique $[\underline{7}, \underline{8}]$. This unfortunate situation has changed owing to the recent extension of the superposition $T$-matrix method (STMM) $[\underline{9}, 10]$ to arbitrarily clustered and nested spherical domains [11]. Hence, the principal objective of this Letter is to perform the first quantitative comparison of scattering and absorption properties of different types of droplet-soot mixtures. We will also analyze to what extent these properties can be reproduced by a popular EMT, viz., the Maxwell-Garnett approximation (MGA) [].

To cover a wide range of potential mixing scenarios, we consider the following 11 models (sketched in Fig. 1) while keeping the wavelength fixed at $\lambda=0.55 \mu \mathrm{m}$.

Model A. A fluffy soot aggregate composed of $N=75$ identical spherical monomers is internally mixed with (i.e., is completely imbedded in [12]) a spherical water droplet such that the distance between the droplet center and the geometrical center of the fractal is equal to $d$. The droplet radius, $R$, is such that the droplet size parameter $X=2 \pi R / \lambda$ is equal to 40 , while the droplet refractive index is 1.33 . The morphology of the soot aggregate is parameterized by the standard statistical scaling law $N=k_{0}\left(R_{g} / r\right)^{D_{f}}[\underline{13}]$, where $r$ is the monomer radius, $k_{0}=1.19$ is the fractal prefactor, $D_{f}=1.82$ is the fractal dimension, and $R_{g}$, called the radius of gyration, is a measure of the overall aggregate radius. The black carbon refractive index is fixed at $1.95+i 0.79$, while the monomer radius is fixed at the value corresponding to the monomer size parameter $x=2 \pi r / \lambda=1$ (note that these numbers do not necessarily represent the most typical experimental or modeling values; cf. [14-16]). All scattering and absorption properties are averaged over the uniform orientation distribution of the resulting inhomogeneous particle.

Model B. As in Model A, but the fluffy soot aggregate collapses into a compact cluster with $D_{f}=3$.

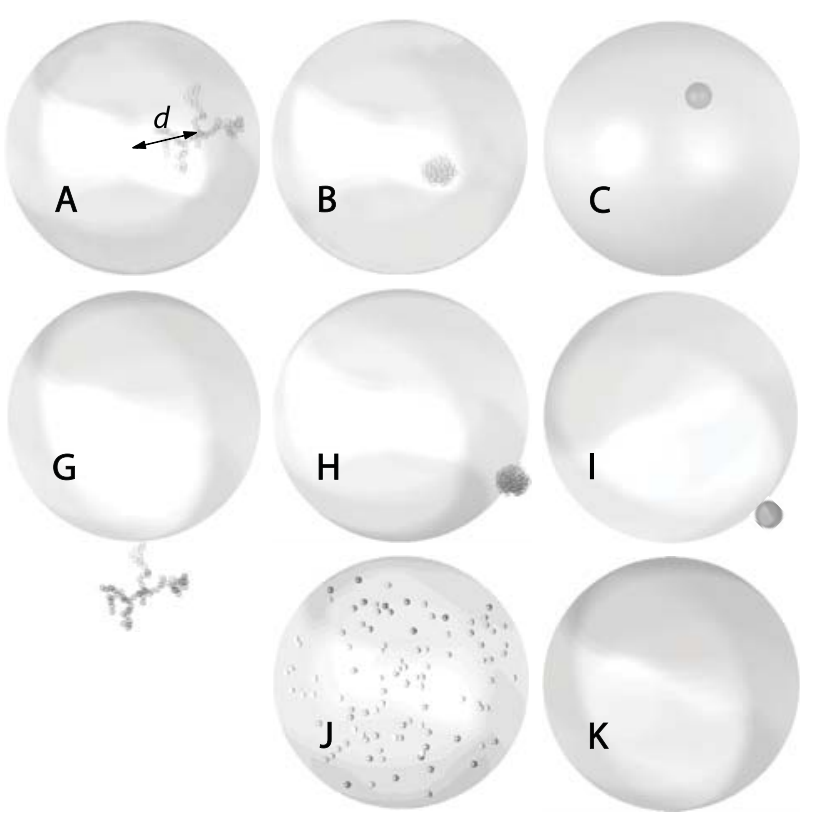

Fig. 1. Models of soot-contaminated water droplets. 
Model C. As in Model B, but the compact soot cluster is replaced by a large homogeneous spherical soot inclusion having the same volume as the soot cluster.

Model D. As in Model A, but the spherical water droplet and the fluffy soot aggregate are externally mixed [12], i.e., are widely separated and act as "independent scatterers". The radius of the droplet is adjusted to keep the combined volume of the water and black carbon fixed, the corresponding droplet size parameter being 39.984. All scattering and absorption properties of the soot cluster are averaged over the uniform orientation distribution.

Model E. As in Model B, but the spherical water droplet and the compact soot aggregate are externally mixed and the radius of the droplet is adjusted. All scattering and absorption properties of the soot cluster are averaged over the uniform orientation distribution.

Model F. As in Model C, but the spherical water droplet and the homogeneous spherical soot particle are externally mixed and the radius of the droplet is adjusted.

Model G. As in Model D, but the fluffy soot aggregate and the droplet are semi-externally mixed [12], i.e., the aggregate is attached externally to the surface of the droplet.

Model H. As in Model E, but the compact soot aggregate and the droplet are semi-externally mixed.

Model I. As in Model F, but the homogeneous spherical soot particle and the droplet are semi-externally mixed.

Model J. As in Model A, but the 75 soot monomers are quasi-randomly distributed throughout the droplet interior rather than form a cluster.

Model K. A homogeneous droplet has a size parameter of 40 , and its refractive index is computed according to the MGA for the same volume of soot material as in Models A-J.

Figure 2 depicts the orientation-averaged absorption cross section, $C_{\text {abs }}$, for Models $\mathrm{A}-\mathrm{C}$ as a function of the size-parameter distance, $2 \pi d / \lambda$. It is obvious that, for the same total amount of black carbon, the cumulative absorption (i) strongly depends on the specific morphology of the soot inclusion and (ii) is maximal when the soot inclusion is located sufficiently far from the droplet boundary. In geometrical optics terms, distancing the inclusion from the boundary and increasing its geometric cross section serves to maximize the probability of the inclusion to "intercept" refracted and internally reflected "rays". This apparently explains both traits visible in Fig. 2, at least qualitatively.

The first trait is further illustrated by the third column of Table 1, which shows the absorption cross section for Models $\mathrm{A}-\mathrm{C}$ averaged over all relevant distances, $d$. This column also demonstrates that absorption becomes minimal when the entire soot volume is concentrated in one homogeneous spherical particle that is mixed with the droplet externally or semi-externally. On the other hand, breaking a soot fractal into individual monomers and distributing them quasi-uniformly throughout the droplet yields the greatest $C_{\text {abs }}$ value. It is quite remarkable that keeping the amount of soot fixed but switching from Model F to Model J serves to increase the specific soot absorption by a factor exceeding 6.5.

Table 1 also lists the respective values of the extinction cross section, $C_{\text {ext }}$, scattering cross section, $C_{\mathrm{sca}}$,
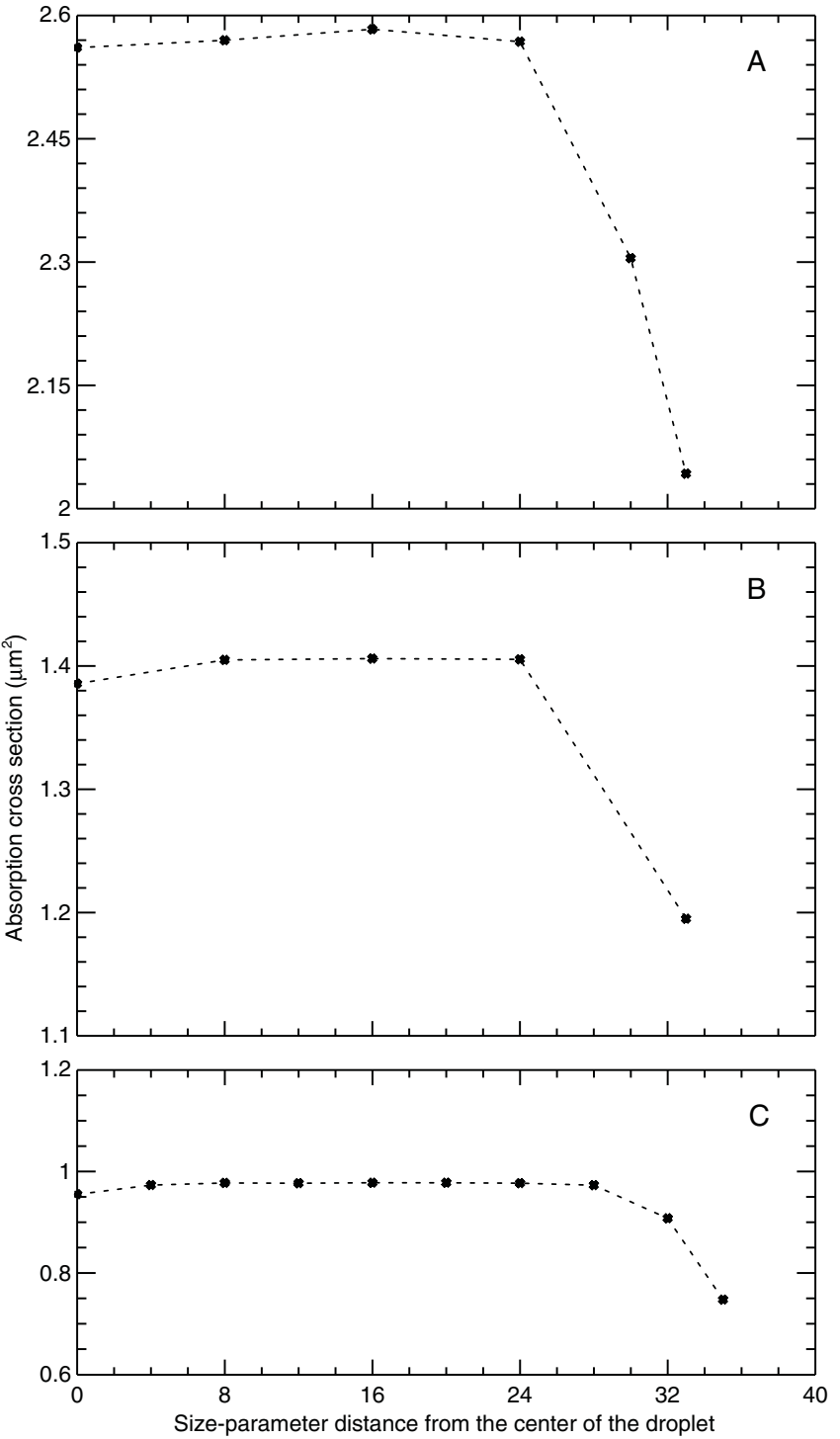

Fig. 2. Absorption cross section versus $2 \pi d / \lambda$ for Models A-C.

asymmetry parameter, $g$, and single-scattering albedo, $\varpi$. It is seen that these integral characteristics are relatively less affected by the soot morphology and type of mixing. However, the single-scattering albedo difference between Models F (or I) and $\mathrm{J}$ is quite significant since it can result in a substantial change of the reflectivity of an

Table 1. Integral Radiative Characteristics of Water Droplets Mixed with Black Carbon

\begin{tabular}{lccccc}
\hline Model & $C_{\text {ext }}\left(\mu \mathrm{m}^{2}\right)$ & $C_{\text {abs }}\left(\mu \mathrm{m}^{2}\right)$ & $C_{\text {sca }}\left(\mu \mathrm{m}^{2}\right)$ & $g$ & $\varpi$ \\
\hline $\mathrm{A}$ & 77.9846 & 2.4649 & 75.5197 & 0.8395 & 0.9684 \\
$\mathrm{~B}$ & 77.3582 & 1.3594 & 75.9988 & 0.8398 & 0.9824 \\
$\mathrm{C}$ & 77.0620 & 0.9443 & 76.1177 & 0.8396 & 0.9877 \\
$\mathrm{D}$ & 79.7094 & 1.5891 & 78.1203 & 0.8362 & 0.9801 \\
$\mathrm{E}$ & 78.4154 & 0.8850 & 77.5304 & 0.8376 & 0.9887 \\
$\mathrm{~F}$ & 77.8879 & 0.5753 & 77.3126 & 0.8370 & 0.9926 \\
$\mathrm{G}$ & 78.6325 & 1.5835 & 77.0489 & 0.8373 & 0.9799 \\
$\mathrm{H}$ & 77.4591 & 0.8757 & 76.5834 & 0.8387 & 0.9887 \\
$\mathrm{I}$ & 77.1285 & 0.5763 & 76.5522 & 0.8380 & 0.9925 \\
$\mathrm{~J}$ & 77.0129 & 3.7469 & 73.2660 & 0.8412 & 0.9513 \\
$\mathrm{~K}$ & 76.6184 & 3.9245 & 72.6939 & 0.8510 & 0.9488 \\
\hline
\end{tabular}


optically thick liquid-water cloud. Indeed, the cloud albedo is largely defined by the so-called single-scattering co-albedo, $1-\varpi$, rather than by $\varpi$. Table XIX on page 340 of [17] shows that switching from Model $\mathrm{F}$ to model J can be expected to reduce the spherical albedo of an optically semi-infinite cloud by almost a factor of 2 . Another important result is that the pairs of external and semiexternal mixing scenarios $(\mathrm{D}, \mathrm{G}),(\mathrm{E}, \mathrm{H})$, and $(\mathrm{F}, \mathrm{I})$ yield nearly identical results.

The last row of Table 1 shows the result of applying the MGA. It is quite obvious that the MGA values of the absorption cross section and single-scattering albedo reproduce reasonably well the STMM results for Model J but not those for all the other models.

The angular distribution and the polarization state of the scattered light can be conveniently described in terms of the normalized Stokes scattering matrix,

$$
\tilde{\mathbf{F}}(\Theta)=\left[\begin{array}{cccc}
a_{1}(\Theta) & b_{1}(\Theta) & 0 & 0 \\
b_{1}(\Theta) & a_{2}(\Theta) & 0 & 0 \\
0 & 0 & a_{3}(\Theta) & b_{2}(\Theta) \\
0 & 0 & -b_{2}(\Theta) & a_{4}(\Theta)
\end{array}\right],
$$

where $\Theta \in\left[0^{\circ}, 180^{\circ}\right]$ is the angle between the incidence and scattering directions [18]. The $(1,1)$ element of $\tilde{\mathbf{F}}(\Theta)$ is the conventional phase function normalized according to

$$
\frac{1}{2} \int_{0}^{\pi} \mathrm{d} \Theta \sin \Theta a_{1}(\Theta)=1 .
$$

Two unique properties of a homogeneous, perfectly spherical particle are the identities

$$
a_{2}(\Theta) / a_{1}(\Theta) \equiv 1 \quad \text { and } \quad a_{3}(\Theta) / a_{4}(\Theta) \equiv 1
$$

Any EMT always reproduces these identities by definition, provided that the surface of the host is perfectly spherical. However, Fig. 3(a) reveals a fundamental violation of the first identity by the numerically exact STMM results and thereby illustrates an essential limitedness of the concept of effective refractive index. The violation of the second identity (not shown) is equally significant. Interestingly, the angular locations of all minima in Fig. 3(a) appear to be the same irrespective of the mixing scenario.

Figures 3(b) and 3(c) show STMM results for an $X=$ 20 droplet quasi-uniformly filled with $N$ soot inclusions, each having a size parameter $x=1$. Fig. 3(b) shows that, with increasing $N$, the deviation of the ratio $a_{2}(\Theta) / a_{1}(\Theta)$ from unity first increases, then saturates, and finally starts to decrease. This behavior can be explained qualitatively by competing effects of increasing "multiple scattering" and increasing absorption. The former serves to reduce the ratio $a_{2}(\Theta) / a_{1}(\Theta)$, whereas the latter serves to suppress the contribution of scattered light emerging from the droplet interior and thereby enhances the
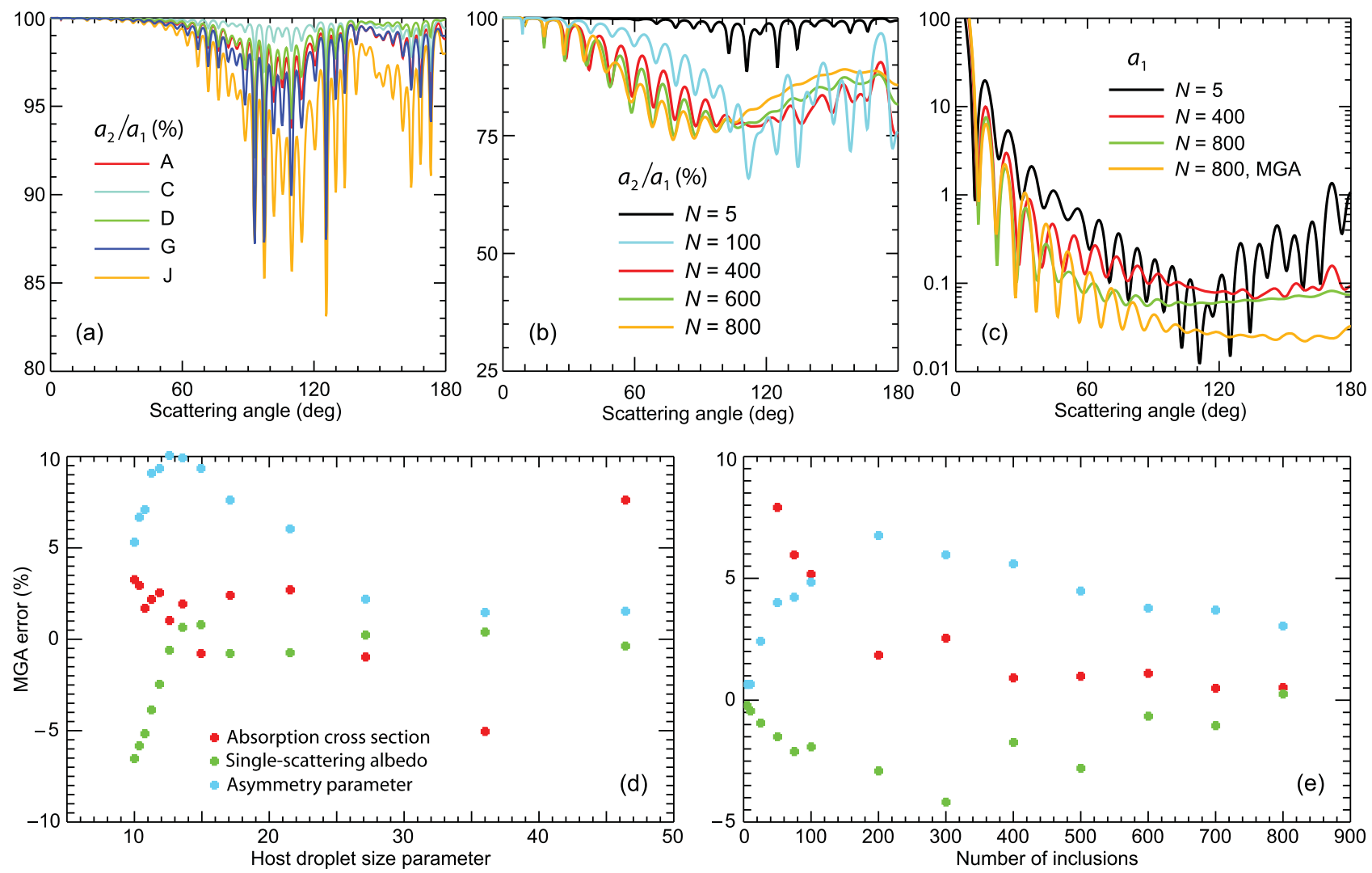

Fig. 3. (a) The ratio $a_{2}(\Theta) / a_{1}(\Theta)$ for Models A, C, D, G, and J. The elements of the normalized scattering matrix are averaged over orientations and, for Models A and C, over the applicable ranges of $d$. (b), (c) STMM and MGA results for a water droplet filled quasiuniformly with $N$ small black carbon inclusions. (d), (e) MGA errors as functions of the droplet size parameter or the number of black carbon inclusions. 
relative contribution of the external reflection satisfying the first identity of Eq. (3). In general, Figs. 3(a) and 3(b) demonstrate the potential of using measurements of the ratio $a_{2}(\Theta) / a_{1}(\Theta)$ as an optical characterization tool highly sensitive to nonspherical and/or inhomogeneous particle morphology (cf. [19,20]).

The phase function results plotted in Fig. 3(c) for the same $X=20$ droplet show that increasing $N$ serves to make $a_{1}(\Theta)$ progressively featureless at side- and back-scattering angles and to suppress the backscattering phase function values. The effect of applying the MGA is qualitatively similar, but results in quantitative errors exceeding a factor of 3 in the case of the droplet with 800 soot inclusions.

Figures 3(d) and 3(e) further quantify MGA errors in integral radiometric characteristics computed for Model $\mathrm{J}$ assuming a size parameter of the soot inclusions fixed at $x=1$. In Fig. $3(\mathrm{~d})$, the droplet size parameter, $X$, is increased from 10 to 46.42 , while the number of inclusions is kept fixed at 100, thereby implying a decrease of the black carbon volume fraction from $10 \%$ to $0.1 \%$. In Fig. 3(e), the droplet size parameter $X$ is fixed at 20 , while the number of inclusions, $N$, grows from 5 to 800 . The black carbon volume fraction thus increases from essentially zero to $10 \%$. It is seen that, in all cases, the MGA errors do not exceed $\sim 10 \%$.

In summary, our numerically exact STMM results reveal a very strong dependence of the soot specific absorption on the mode of soot-water mixing in liquid-water clouds contaminated by black carbon. The absorption is maximized when the soot material gets quasi-uniformly distributed throughout the droplet interior in the form of numerous small monomers (Model $J)$. The absorption is minimal when black carbon exists in the form of larger homogeneous particles mixed with cloud droplets externally or semi-externally (Models F and I). Similar conclusions were reached in a recent modeling study of mixtures of soot aggregates with small sulfate aerosols based on the discrete dipole approximation [21]. The presence of soot has other noticeable manifestations, such as its effects on the single-scattering co-albedo and the elements of the scattering matrix. It is beyond the scope of this Letter to discuss the relative plausibility of the mixing scenarios represented by Models A-J. However, the morphological range captured by these scenarios implies a wide range of remote sensing and radiation budget implications of the presence of black carbon in liquid-water clouds.

An important by-product of our study is the first quantitative analysis of the accuracy of the MGA as applied to micrometer-sized water droplets mixed with black carbon. It appears that the only worthwhile application of the MGA is the calculation of the optical cross sections, single-scattering albedo, and asymmetry parameter for the quasi-uniform mixing scenario described by Model J.

This material is based upon work supported by the NASA Remote Sensing Theory Program managed by Lucia Tsaoussi and the NASA Radiation Sciences Program managed by Hal Maring. The majority of numerical results were obtained with the "Discover" supercomputer at the NASA Center for Climate Simulation.

\section{References}

1. P. Chýlek, G. B. Lesins, G. Videen, J. G. D. Wong, R. G. Pinnick, D. Ngo, and J. D. Klett, J. Geophys. Res. 101, 23365 (1996).

2. J. Li, E. Mlawer, and P. Chýlek, J. Geophys. Res. 116, D23212 (2011).

3. Z. Wang, H. Zhang, J. Li, X. Jing, and P. Lu, J. Geophys. Res. 118, 3662 (2013).

4. M. I. Mishchenko, J. W. Hovenier, W. J. Wiscombe, and L. D. Travis, in Light Scattering by Nonspherical Particles: Theory, Measurements, and Applications, M. I. Mishchenko, J. W. Hovenier, and L. D. Travis, eds. (Academic, 2000), pp. 29-60.

5. F. M. Kahnert, J. Quant. Spectrosc. Radiat. Transfer 79-80, 775 (2003).

6. P. Chýlek, G. Videen, D. J. W. Geldart, J. S. Dobbie, and H. C. W. Tso, in Light Scattering by Nonspherical Particles: Theory, Measurements, and Applications, M. I. Mishchenko, J. W. Hovenier, and L. D. Travis, eds. (Academic, 2000), pp. 273-308.

7. M. I. Mishchenko and A. Macke, J. Quant. Spectrosc. Radiat. Transfer 57, 767 (1997).

8. L. Liu, M. I. Mishchenko, S. Menon, A. Macke, and A. A. Lacis, J. Quant. Spectrosc. Radiat. Transfer 74, 195 (2002).

9. D. W. Mackowski and M. I. Mishchenko, J. Opt. Soc. Am. A 13, 2266 (1996).

10. D. W. Mackowski and M. I. Mishchenko, J. Quant. Spectrosc. Radiat. Transfer 112, 2182 (2011).

11. D. W. Mackowski, J. Quant. Spectrosc. Radiat. Transfer 133, 264 (2014).

12. M. I. Mishchenko, L. Liu, L. D. Travis, and A. A. Lacis, J. Quant. Spectrosc. Radiat. Transfer 88, 139 (2004).

13. C. M. Sorensen, Aerosol Sci. Technol. 35, 648 (2001).

14. T. C. Bond and R. W. Bergstrom, Aerosol Sci. Technol. 40, 27 (2006).

15. K. Adachi, S. H. Chung, and P. R. Buseck, J. Geophys. Res. 115, D15206 (2010).

16. M. Kahnert, Aerosol Sci. Technol. 44, 453 (2010).

17. E. G. Yanovitskij, Light Scattering in Inhomogeneous Atmospheres (Springer, 1997).

18. M. I. Mishchenko, L. D. Travis, and A. A. Lacis, Scattering, Absorption, and Emission of Light by Small Particles (Cambridge University, 2002).

19. M. I. Mishchenko, L. Liu, and D. W. Mackowski, J. Quant. Spectrosc. Radiat. Transfer 123, 135 (2013).

20. M. I. Mishchenko, L. Liu, and D. W. Mackowski, Opt. Lett. 39, 1701 (2014).

21. M. Kahnert, T. Nousiainen, and H. Lindqvist, Opt. Express 21, 7974 (2013). 